\title{
DIGEST Scale Predictis More Quality of Life Than PAS: The Residue Influence on Supracricoid Laryngectomy
}

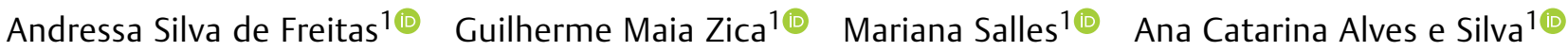 \\ Thiago Huaytalla Silva ${ }^{2(0)}$ Fernando Luiz Dias ${ }^{1}{ }^{(0)}$ Izabella Costa Santos ${ }^{1}$
}

\footnotetext{
${ }^{1}$ Departamento de Cirurgia de Cabeça e Pescoço, Instituto Nacional do Câncer, Ministério da Saúde, Rio de Janeiro, RJ, Brazil

2 Departamento de Nutrição e Dietética, Universidade Federal do Rio de Janeiro, Rio de Janeiro, RJ, Brazil
}

Address for correspondence Andressa Silva de Freitas, PhD, Seção de Cirurgia de Cabeça e Pescoço, Instituto Nacional do Câncer (INCA), Praça da Cruz Vermelha, 23, Centro - Rio de Janeiro (RJ), CEP 20230130, Brasil (e-mail: andressa.freitas@inca.gov.br).

Int Arch Otorhinolaryngol 2022;26(3):e357-e364.

\begin{abstract}
Keywords

- rehabilitation

- radiology

- deglutition disorders

- laryngectomy

- quality of life

Introduction Supracricoid laryngectomy (SCL CHEP) removes $\sim 70 \%$ of the larynx, resulting in structural rearrangement and modification of the swallowing mechanism, promoting chronic dysphagia. One of the consequences of this new physiology is the formation of pharyngeal residues that can increase the possibility of aspiration. The formation of residues after SCL CHEP, its functional consequences, and its influence on quality of life $(\mathrm{QOL})$ is still poorly described in the literature.

Objective To investigate and compare the association between self-reported QoL and objective assessments of swallowing function in patients undergoing SCL CHEP.

Methods A cross-sectional study was performed from 2018 to 2020 in a reference service for head and neck surgery in Brazil. A total of 860 swallowing videofluoroscopy images were evaluated using the Penetration and Aspiration Scale (PAS) and Dynamic Imaging Grade of Swallowing Toxicity (DIGEST).

Results In a group of 86 patients, there was a significant relationship between oncological staging and the global $(p<0.001)$ and total $(p=0.002)$ QoL domains. There was a negative correlation between the DIGEST scale and the emotional domain of the QoL protocol $(p=0.045)$. The swallowing function proved to be relevant for QoL. Conclusion The PAS scale did not show any correlation with QoL. The functional performance of swallowing according to the DIGEST scale was coherent with the QOL scores. It is suggested that the residue may be a more relevant aspect for QoL than the aspiration, making DIGEST a promising tool in the assessment of dysphagic patients.
\end{abstract}

Study performed at the Interdisciplinary Head and Neck Laboratory (Laboratório Interdisciplinar de Cabeça e Pescoço - LICEP) of the National Cancer Institute (Instituto Nacional de Câncer - INCA), Ministry of Health, Rio de Janeiro, RJ, Brazil. received

June 5,2020

accepted

January 7, 2021

published online

November 3, 2021
DOI https://doi.org/ 10.1055/s-0041-1730306. ISSN 1809-9777. (c) 2021. Fundação Otorrinolaringologia. All rights reserved.

This is an open access article published by Thieme under the terms of the Creative Commons Attribution-NonDerivative-NonCommercial-License, permitting copying and reproduction so long as the original work is given appropriate credit. Contents may not be used for commercial purposes, or adapted, remixed, transformed or built upon. (https://creativecommons.org/ licenses/by-nc-nd/4.0/)

Thieme Revinter Publicações Ltda., Rua do Matoso 170, Rio de Janeiro, RJ, CEP 20270-135, Brazil 


\section{Introduction}

Supracricoid laryngectomy (SCL) is an open partial surgery indicated for tumors in moderately advanced stages and has significantly reduced the indications for surgeries with a higher degree of mutilation, such as total laryngectomy. ${ }^{1-3}$ It is a procedure that can also be indicated for rescue surgeries, with good cancer control. ${ }^{4}$

Supracricoid laryngectomy consists of the removal of the pedicle from the epiglottis, thyroid cartilage, laryngeal ventricles, vocal folds, ventricular bands, of the paraglottic space and the pre-epiglottic space. To enable the formation of the neoglottis, one or both cricoarytenoid units, the epiglottis and cricoid, must remain. Surgical reconstruction occurs, among variations, through cricohioidoepiglotopexia (CHEP), in which a suture is performed between the remaining structures (cricoid cartilage, epiglottis and hyoid bone)., Cricohioidoepiglotopexia elevates the laryngeal complex to the level of the hyoid bone, causing a structural rearrangement in the pharyngeal recesses and modification of the swallowing process. 6,7

Supracricoid laryngectomy CHEP can be classified as a type Ila partial open horizontal laryngectomy, according to the nomenclature proposed in 2014 by the European Laryngological Society. ${ }^{4,8}$ The main functional consequence is a chronic sequel in the closure of the airways that leads to dysphonia and dysphagia. 1,2,7,8 Dysphagia, due to its potential to cause greater morbidity and morbidity, generally receives greater clinical and scientific attention. Many authors have described persistent aspiration in the postoperative period, a fact that generates attention on the longterm consequences of this chronic dysfunction. ${ }^{8,9}$ Dysphagia may be associated with a higher risk of pneumonia, less oral intake, more time to use a nasogastric tube (NGT), weight loss, changes in social activities and, consequently, worse quality of life (QoL). 2,3,7,9

An experienced multidisciplinary team is necessary so that the patient can have an adequate clinical course with the adequacy of the swallowing function from 3 to 6 months after surgery. Despite this consensus, it is known, through imaging exams, that the safety and efficiency of swallowing are severely compromised. It is possible to detect long-term chronic aspiration, especially with liquids, and the formation of residues after swallowing. 7,9

There are different methods to assess dysphagia and its impact, including clinical, instrumental, and QoL protocols. $^{2,9}$ Finding an ideal measure for research purposes is challenging due to the complex physiology of swallowing and its association with food and diet. In recent years, QoL has become an increasingly important parameter in cancer treatment decisions. In patients with head and neck cancer, treatment can have a profound impact on QoL. ${ }^{10}$ Therefore, long-term QoL has become an important endpoint, along with conventional survival endpoints. ${ }^{11}$

Currently, videofluoroscopy is the gold-standard test to assess swallowing dynamics. ${ }^{9}$ The Penetration and Aspiration Scale (PAS) was developed in 1996 by Rosenbek et al. in an attempt to categorize the severity of dysphagia, through the identification of aspiration and penetration in the videofluoroscopic swallowing study (VFSS). ${ }^{12}$ Swallowing analysis was based on the description of the presence or absence of aspiration for years in numerous studies, and safety was the only indication of good swallowing. ${ }^{13}$

Recently, the study of dysphagia has turned to other aspects besides safety. Currently, it is known that efficiency can have a negative impact equal to or greater than safety (penetration and aspiration) in functional and QoL criteria. Aspiration in late post-SCL individuals usually occurs in a chronic and silent form and, therefore, does not present itself as a common complaint or with relevance in its oral intake. ${ }^{7-9}$ The presence of residue (swallowing efficiency), resulting from structural changes in the surgery, makes it difficult to progress food consistency, promotes the sensation of stopped food, suffocation, discomfort and damages QoL. ${ }^{9,14}$

Unfortunately, residue analysis is still a dilemma in the VFSS from the three-dimensional (3D) characteristic of pharyngeal recesses. Some studies have attempted to assess the presence of the residue, with methodological heterogeneity and impaired reproducibility. ${ }^{9,14-17}$

Meyers et al., in 2017, described QoL in a heterogeneous group of 168 head and neck cancer survivors, and concluded that aspiration and residue show independent effects. The authors describe that the residue has a greater impact on QoL than the aspiration event, standing out as a primary measure of swallowing function. Compared with other methods, the measurement of the residue in this study also has a difficult reproducibility. ${ }^{14}$

Specifically in SCL-CHEP, there are reports in the literature that demonstrate a good overall QoL. ${ }^{3,9}$ In a 2018 study, Pizzorni et al. used Fiberscope Endoscopic Evaluation Swallowing (FEES) in an attempt to understand the association between swallowing function and QoL after SCL-CHEP. They describe that QoL was significantly affected by the severity of aspiration; however, they conclude that the perception of the patient was not sufficient to detect the severity of his dysphagia. This finding suggests that there may be an incompatibility between the assessment technique and the impact of the function on the patient. $^{9}$

The formation of residues after SCL-CHEP and its consequences is still poorly described. In a recent 2019 study, there is a report that the formation of the residue is justified by increased pharyngeal transit time, reduced opening of the upper esophageal sphincter, and incomplete retraction of the base of the tongue. ${ }^{7}$ There are still no studies that assess the influence of residue on QOL.

In 2017, Hutcheson et al. developed a new tool for analyzing the VFSS. The Dynamic Imaging Grade of Swallowing Toxicity (DIGEST) scale offers an ordinal summary of five points to standardize the report and description of the bolus passage through the pharynx, and brings an assessment that considers safety, using PAS, and efficiency, through analysis of the residue in the VFSS exam. ${ }^{15}$ It is a validated tool to assess the severity of pharyngeal dysphagia, which assigns a value by correlating the parameters of safety and efficiency of swallowing. No studies were found using this new tool related to aspects of QoL. 
The aim of the present study was to analyze the association between QoL and changes in the safety and efficiency of swallowing detected by the VFSS in patients undergoing SCLCHEP. Based on the premise that efficiency is more relevant to QoL than safety, the strategy of finding new tools that contemplate the impact of dysphagia in a multidimensional way has become essential.

\section{Methods}

Cross-sectional study, approved by the Research Ethics Committee of the institution under CAAE: 26331314.2.0000.5274, 616.249. Individuals of both sexes submitted to SCL-CHEP, from the Instituto Nacional de Câncer (Brazilian National Cancer Institute), were included. Exclusion criteria were patients $<18$ years old, uncooperative due to linguistic-cognitive impairments, and undergoing another type of surgical intervention in the laryngeal region after SCL-CHEP. All volunteers participating in the present study signed the consent form.

The individuals eligible for the present study were located in the service from 2018 to 2020. Demographic and clinical information were collected through the analysis of physical and electronic medical records. For speech therapy and QoL assessments, the patients were approached and invited to participate in the study and to be submitted to the procedures described below.

\section{Swallowing Assessment Instruments and Procedures} The VFSS was used to evaluate the swallowing of the research patients. A total of 860 exams were performed using the Siemens AXIOM Iconos Remote Control X-ray equipment (serial number 13020) (Siemens, Munich, Germany) and were evaluated using a Logemann-based protocol from 1998. ${ }^{18}$ All video segments were recorded in the side view plane, with a capture rate of 30 frames per second.

The contrast was offered in a cup, using 100\% dilutions of Bariogel (Cristália, Itapira, São Paulo, Brazil) barium sulfate (BS), mineral water and the ThickenUp Clear Thickener Resource (Nestlé Health Science, São Bernardo do Campo, São Paulo, Brazil). Three consistencies were evaluated: thin liquid in $5 \mathrm{~mL}$ ( $2.5 \mathrm{~mL}$ of water $+2.5 \mathrm{~mL}$ of BS), $10 \mathrm{~mL}$ ( $5 \mathrm{~mL}$ of water $+5 \mathrm{~mL}$ of $\mathrm{BS})$ and $20 \mathrm{~mL}(10 \mathrm{~mL}$ of water $+10 \mathrm{~mL}$ of BS); semi-liquid consistency in $5 \mathrm{~mL}$ of BS, $10 \mathrm{~mL}$ of BS and $20 \mathrm{~mL}$ of BS; and pasty consistency in $5 \mathrm{~mL}$ ( $5 \mathrm{~mL}$ of BS $+1.2 \mathrm{~g}$ of thickener), $10 \mathrm{~mL}$ ( $10 \mathrm{~mL}$ of $\mathrm{BS}+2.4 \mathrm{~g}$ of thickener) and $20 \mathrm{~mL}$ ( $20 \mathrm{~mL}$ of $\mathrm{BS}+3.6 \mathrm{~g}$ of thickener). To standardize the exam, solid consistency was not included, since some individuals have dental absences. The PAS ${ }^{12}$ and the DIGEST ${ }^{15}$ were used to analyze the VFSS.

The DIGEST is a validated staging tool to assess the severity of pharyngeal dysphagia based on the study of the VFSS. The scale first assigns two component scores: 1) safety rating, and 2) efficiency rating. To classify safety, the evaluator must assign the PAS scale score observed during the swallowing of different consistencies of the bolus, according to the VFSS protocol performed. ${ }^{12,18}$ To estimate the efficiency rating, the evaluator assigns a maximum percentage of pharyngeal residue on an ordinal scale ( $<10 \%$; 10 to $49 \%$; 50 to $90 \%$; and $>90 \%$ ), to assign a residue pattern after the first swallow. The DIGEST offers an ordinal summary of 5 points that assigns a value by correlating the parameters of safety and efficiency of swallowing: grade $0=$ without dysphagia; $1=$ mild dysphagia; $2=$ moderate dysphagia; $3=$ severe dysphagia; $4=$ life-threatening. ${ }^{15}$

\section{Quality of Life Assessment}

The only tool available to assess the effects of swallowing changes on QoL in individuals undergoing treatment for head and neck cancer is the M. D. Anderson Dysphagia Questionnaire (MDADI), developed and validated by Chen et al. in $2001 .^{19}$ The tool was validated for Brazilian Portuguese by Guedes et al. in $2013^{20}$ and consists of 20 questions, on the global, emotional, functional and physical domains. All scales have 5 possible responses, ranging from 1 (strongly agree) to 5 (strongly disagree), except 1 item from the emotional subscale (E7) and 1 item from the functional subscale (F2), which are scored varying from 5 (strongly agree) to 1 (strongly disagree). The score for each subscale is obtained by calculating the average score of its items, multiplied by 20 . The total score is obtained from the average of the scores for each domain (emotional, functional and physical) multiplied by 20 . The individual can obtain a score ranging from 0 to 100 , and the lower the value of the observed score, the worse the effect of dysphagia in their QOL and functionality. ${ }^{2,19}$

\section{Statistical Analysis}

The data were analyzed using STATA software, version 13.0 (StataCorp, College Station, TX, USA). Quantitative variables were described as mean and standard deviation (SD) or median and minimum and maximum, and qualitative variables were expressed in absolute and relative frequency. The normality of the data was analyzed using the histogram and the Kolmogorov-Smirnov test. The Student $t$-test and analysis of variance (ANOVA) were applied to compare means, and Pearson linear correlation was applied to verify a possible correlation between variables. The significance level of $5 \%$ $(p<0.05)$ was adopted for all statistical tests.

\section{Results}

A total of 86 patients were evaluated, with a mean age of 61.4 $( \pm 9.55)$ years old and a median of 62 years old, ranging from 28 to 82 years old. There was a higher prevalence of males ( $n=84 ; 97.67 \%)$, married $(n=61 ; 70.93 \%)$, with low education (up to elementary school) $(n=52 ; 60.47 \%)$, smokers $(n=72 ; 83.72 \%)$ and alcoholics $(n=71 ; 82.56 \%)$ at diagnosis and with intermediate or advanced stage laryngeal tumors ( $n=82 ; 95.35 \%)$ of histopathological squamous cell carcinoma $(n=85 ; 98.83 \%)$ (-Table 1 ).

All individuals underwent SCL-CHEP; most of them had 2 arytenoids in their neoglot $(n=57 ; 66.28 \%), \sim 71 \%(n=61)$ underwent adjuvant radiotherapy, only 3 patients (3.48\%) presented wound infections in the postoperative period, and $1(1.16 \%)$ patient presented cervical fistula. There was no 
Table 1 Characterization of 86 patients undergoing supracricoid laryngectomy with reconstruction using cricohioidoepiglotopexia

\begin{tabular}{|c|c|c|c|}
\hline \multicolumn{2}{|l|}{ Variables } & \multirow{2}{*}{$\begin{array}{l}\mathrm{N} \\
61.4(9.55)\end{array}$} & \multirow{2}{*}{$\begin{array}{l}\% \\
62(28-82)\end{array}$} \\
\hline Age (years old) ${ }^{a, b}$ & & & \\
\hline \multirow[t]{2}{*}{ Sex } & Female & 2 & 2.32 \\
\hline & Male & 84 & 97.67 \\
\hline \multirow[t]{2}{*}{ Marital status } & Single & 25 & 29.07 \\
\hline & Married & 61 & 70.93 \\
\hline \multirow[t]{2}{*}{$\begin{array}{l}\text { Educational } \\
\text { level }\end{array}$} & $\begin{array}{l}\text { Elementary } \\
\text { School }\end{array}$ & 52 & 60.47 \\
\hline & $\begin{array}{l}\text { High school } \\
\text { and higher } \\
\text { education }\end{array}$ & 34 & 39.53 \\
\hline \multirow[t]{2}{*}{ Smoker } & Yes & 72 & 83.72 \\
\hline & No & 14 & 16.28 \\
\hline \multirow{2}{*}{$\begin{array}{l}\text { Alcohol } \\
\text { consumption }\end{array}$} & Yes & 71 & 82.56 \\
\hline & No & 15 & 17.44 \\
\hline \multirow[t]{2}{*}{ Arytenoid } & 1 & 29 & 33.72 \\
\hline & 2 & 57 & 66.28 \\
\hline \multirow{3}{*}{$\begin{array}{l}\text { Oncological } \\
\text { staging }\end{array}$} & II & 53 & 61.63 \\
\hline & III & 29 & 33.72 \\
\hline & IV & 4 & 4.65 \\
\hline \multirow{2}{*}{$\begin{array}{l}\text { Adjuvant } \\
\text { radiotherapy }\end{array}$} & Yes & 61 & 70.93 \\
\hline & No & 25 & 29.07 \\
\hline$\%$ Emotional $^{\mathrm{b}}$ & & 100 & $(46.65-100)$ \\
\hline$\%$ Functional $^{\mathrm{b}}$ & & 100 & $(32-100)$ \\
\hline \% Physical ${ }^{\mathrm{b}}$ & & 83.75 & $(47.5-100)$ \\
\hline$\%$ Global $^{\text {b }}$ & & 100 & $(20-100)$ \\
\hline$\%$ Total $^{\mathrm{b}}$ & & 94.60 & $(45.12-100)$ \\
\hline DIGEST ${ }^{b}$ & & 2 & $(1-4)$ \\
\hline PAS $^{b}$ & & 5 & $(2-8)$ \\
\hline
\end{tabular}

Abbreviations: DIGEST, Dynamic Imaging Grade of Swallowing Toxicity; $\mathrm{N}$, number; \%, frequency; PAS, Penetration- Aspiration Scale.

$\mathrm{a}=$ Mean / standard deviation

$\mathrm{b}=$ Median / minimum and maximum.

necrosis or hemorrhage, no patient had pneumonia at the time of the study.

There is no permanence of NGT in the evaluated group, that is, all had exclusive orally food and hydration at the time of the evaluation. Only 3 patients remained with a tracheostomy (3.48\%).

The QoL in swallowing, obtained through the MDADI questionnaire, was presented with medians of values $>80$ in all domains. The emotional, functional and global domains obtained the best medians of the scores, presenting the maximum value. The lowest median value was in the physical domain. The emotional domain had a mean of 96.09 ( \pm 9.35 ), and a median of 100 , with a range of 46.65 to 100 . The functional domain had a mean of $92.36( \pm 12.15)$, and a
Table 2 Distribution of the domains of the M. D. Anderson Dysphagia Questionnaire (MDADI - M. D. Anderson Dysfaghia Inventory) in relation to the swallowing functional performance represented by the DIGEST and PAS scales

\begin{tabular}{|l|l|l|l|l|}
\hline $\begin{array}{l}\text { Domains } \\
\text { MDADI }\end{array}$ & \multicolumn{2}{|l|}{ DIGEST } & \multicolumn{2}{l|}{ PAS } \\
\hline & $\mathrm{r}$ & $p$-value & $\mathrm{r}$ & $p$-value \\
\hline Emotional & -0.216 & 0.045 & 0.057 & 0.605 \\
\hline Functional & -0.083 & 0.446 & 0.142 & 0.191 \\
\hline Physical & -0.171 & 0.116 & -0.061 & 0.578 \\
\hline Global & -0.141 & 0.196 & -0.021 & 0.846 \\
\hline Total & -0.180 & 0.097 & 0.031 & 0.774 \\
\hline
\end{tabular}

Abbreviations: DIGEST, Dynamic Imaging Grade of Swallowing Toxicity; PAS, Penetration- Aspiration Scale.

*Pearson correlation.

median of 100 , with a range of 32 to 100 . The physical domain had an average of $82.32( \pm 11.59)$, and a median of 100 with a range of 47.5 to 100 . The global domain had an average of $92.55( \pm 17.09)$, and a median of 100 with a range of 20 to 100 . The total domain had a mean of $89.33( \pm 10.92)$, and a median of 100 with a range from 45.12 to 100 (-Table 1).

The most prevalent classification on the DIGEST scale was DIGEST 2 ( $n=45 ; 52.32 \%)$, followed by the DIGEST 1 ( $n=30$; $34.88 \%), 3(n=9 ; 10.46 \%)$ and $4(n=2 ; 2.32 \%)$. In the PAS scale, the classification with the highest appearance was PAS $5(n=46 ; 53.48 \%)$, followed by number $8(n=25 ; 29.06 \%), 4$ $(n=14 ; 16.27 \%)$ and $2(n=1 ; 1.16 \%)$.

- Table 2 indicates the correlation between the domains of the MDADI questionnaire and the DIGEST and PAS scales. It was possible to observe a negative correlation between the DIGEST and the emotional domain of the QoL protocol $(p=0.045)$, that is, the higher the QoL score, the lower the DIGEST classification.

- Table 3 describes the statistical results of the association between clinical and sociodemographic variables, the functional swallowing results from the PAS and DIGEST scales, and the global and total domain of the MDADI questionnaire. There was a significant relation between staging and the domains of global $(p<0.001)$ and total QoL $(p=0.002)$, respectively. Nonsmokers report better global $(p=0.075)$ and total QoL. Patients with 2 arytenoids had a lower degree of dysphagia when compared with patients with 1 arytenoid (PAS; $p=0.070$ ).

-Figs. 1 and $\mathbf{2}$ were obtained by analyzing all the domains of the MDADI questionnaire in relation to each classification in the functional scales of swallowing PAS and DIGEST. It is possible to visualize the influence of swallowing functionality under the aspect of severity of each scale and its impact on the QOL of individuals.

It is observed in - Fig. 1 that the lowest value of total QoL was found in PAS 4 with an average value of 88.57 (median of 93.15; $\mathrm{SD}=9.21$ ), ranging from 51.83 to 97.5 ; and the highest total QoL was at PAS 2 of 96.5 , in which only 1 patient was present. The lowest QoL value in all PAS classifications was 
Table 3 Association of clinical and sociodemographic variables, functional swallowing results, and the global and total domain of the M. D. Anderson Dysphagia Questionnaire (MDADI - M. D. Anderson Dysfaghia Inventory)

\begin{tabular}{|c|c|c|c|c|c|c|c|c|}
\hline \multirow[t]{2}{*}{ Variables } & \multicolumn{2}{|l|}{ DIGEST } & \multicolumn{2}{|l|}{ PAS } & \multicolumn{2}{|l|}{ Global } & \multicolumn{2}{|l|}{ Total } \\
\hline & Average (SD) & p-value & Average (SD) & p-value* & $\begin{array}{l}\text { Average } \\
\text { (SD) }\end{array}$ & p-value* & $\begin{array}{l}\text { Average } \\
\text { (SD) }\end{array}$ & $p$-value \\
\hline \multicolumn{9}{|l|}{ Age (years old) } \\
\hline$<60$ & $1.76(0.60)$ & 0.696 & $5.73(1.52)$ & 0.773 & $91.76(17.83)$ & 0.730 & $90.53(11.45)$ & 0.742 \\
\hline$\geq 60$ & $1.82(0.78)$ & & $5.63(1.61)$ & & $93.02(16.75)$ & & $89.72(10.78)$ & \\
\hline \multicolumn{9}{|l|}{ Sex } \\
\hline Male & $1.80(0.71)$ & 0.102 & $5.67(1.55)$ & 0.768 & $92.38(17.26)$ & 0.536 & $89.88(11.07)$ & 0.399 \\
\hline Female & $2.00(1.41)$ & & $6.00(2.83)$ & & $100(0.00)$ & & $96.56(1.33)$ & \\
\hline \multicolumn{9}{|l|}{ Marital status } \\
\hline Single & $2.00(0.82)$ & 0.102 & $6.12(1.59)$ & 0.092 & $89.60(20.10)$ & 0.307 & 87.59 (13.37) & 0.188 \\
\hline Married & $1.72(0.66)$ & & $5.49(1.53)$ & & $93.77(15.72)$ & & $91.04(9.80)$ & \\
\hline \multicolumn{9}{|l|}{ Educational level } \\
\hline Elementary School & $1.79(0.61)$ & 0.825 & $5.63(1.53)$ & 0.773 & 90.77 (19.59) & 0.232 & $89.32(12.42)$ & 0.455 \\
\hline $\begin{array}{l}\text { High school and } \\
\text { higher education }\end{array}$ & $1.82(0.87)$ & & $5.73(1.64)$ & & $95.29(12.12)$ & & $91.14(8.38)$ & \\
\hline \multicolumn{9}{|l|}{ Smoker } \\
\hline Yes & $1.76(0.66)$ & 0.262 & $5.60(1.52)$ & 0.303 & $91.11(18.35)$ & 0.075 & $89.15(11.68)$ & 0.088 \\
\hline No & $2.00(0.96)$ & & $6.07(1.77)$ & & $100(0.00)$ & & $94.62(4.13)$ & \\
\hline \multicolumn{9}{|l|}{$\begin{array}{l}\text { Alcohol } \\
\text { consumption }\end{array}$} \\
\hline Yes & $1.85(0.72)$ & 0.110 & $5.77(1.57)$ & 0.199 & 92.39 (17.36) & 0.848 & 89.99 (11.14) & 0.924 \\
\hline No & $1.53(0.64)$ & & $5.20(1.52)$ & & 93.33 (16.33) & & $90.29(10.58)$ & \\
\hline \multicolumn{9}{|l|}{ Arytenoid } \\
\hline 1 & $1.97(0.78)$ & 0.133 & $6.10(1.52)$ & 0.070 & $94.48(15.94)$ & 0.460 & 91.32 (9.09) & 0.442 \\
\hline 2 & $1.71(0.67)$ & & $5.46(5.04)$ & & $91.58(17.71)$ & & 89.39 (11.85) & \\
\hline \multicolumn{9}{|l|}{$\begin{array}{l}\text { Adjuvant } \\
\text { radiotherapy }\end{array}$} \\
\hline Yes & $1.92(0.81)$ & 0.332 & $5.59(1.53)$ & 0.439 & 96.80 (12.49) & 0.142 & $92.69(9.47)$ & 0.153 \\
\hline No & $1.75(0.67)$ & & $5.88(1.67)$ & & $90.82(18.47)$ & & $88.95(11.45)$ & \\
\hline \multicolumn{9}{|l|}{$\begin{array}{l}\text { Oncological } \\
\text { staging }\end{array}$} \\
\hline II & $1.71(0.69)$ & 0.777 & $5.57(1.63)$ & 0.121 & 95.09 (11.02) & $<0.001$ & $91.12(8.27)$ & 0.002 \\
\hline III & $2.00(0.75)$ & & $6.00(1.49)$ & & $89.65(23.07)$ & & 90.07 (12.19) & \\
\hline IV & $1.50(0.58)$ & & $4.75(5.00)$ & & $80(28.28)$ & & $75.47(23.32)$ & \\
\hline
\end{tabular}

Abbreviations: DIGEST, Dynamic Imaging Grade of Swallowing Toxicity; PAS, Penetration- Aspiration Scale; SD, standard deviation.

*Student $t$-test for dichotomous variables and ANOVA for nondichotomous variables.

present in the physical domain, with a median of 90 in PAS 2; 77.5 in PAS 4; 85 in PAS 5; and 82.5 in PAS 8. The highest QoL value in all PAS classifications was present in the functional domain with a median of 96 in PAS 2; 100 in PAS 4; 98 in PAS 5; and 100 in PAS 8. It is not possible to observe in - Fig. 1 a progression in increasing QoL according to the best performance in the PAS swallowing scale.

It is possible to observe in - Fig. 2 a progression of QoL according to the best performance on the DIGEST swallowing scale; the better the functional result in this scale, the better the result of the MDADI questionnaire. The lowest value of total QoL was found in DIGEST 4, with an average value of 71.62 (median of 71.60; $\mathrm{SD}=14.8$ ). The best average total quality of life in DIGEST 1 was 94.66 (median 95.5; SD=6.88), ranging from 51.83 to 100 . The lowest QoL value in all classifications of the DIGEST was present in the physical domain, with a median of 83.75 in DIGEST 1; 85 in DIGEST 2; 82.5 in DIGEST 3; and 62.5 in DIGEST 4. The highest QoL value in all DIGEST classifications was present in the functional domain, with a median of 100 in DIGEST 1; 100 in DIGEST 2; 96 in DIGEST 3; and 84 in DIGEST 4. 

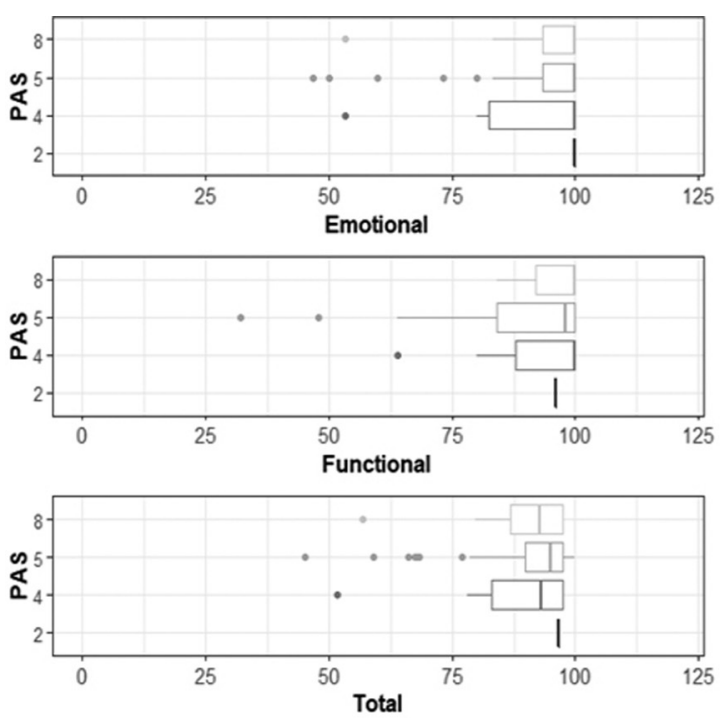
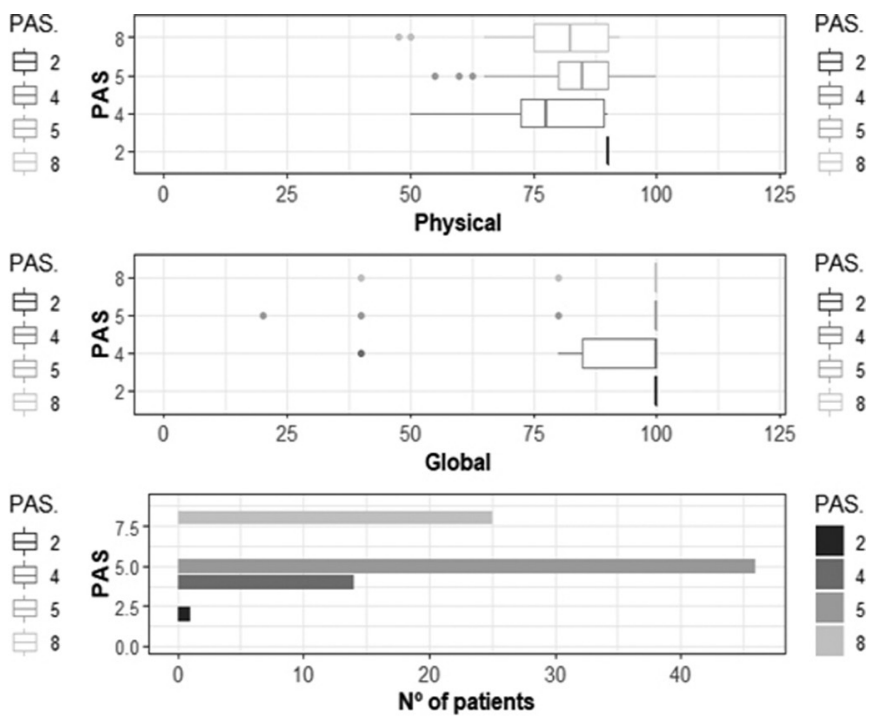

Fig. 1 Boxplot of the distribution by domain of the Swallowing Quality of Life Questionnaire M. D. Anderson Dysfaghia Inventory (MDADI) in relation to each classification from the Penetration and Aspiration Scale (PAS).
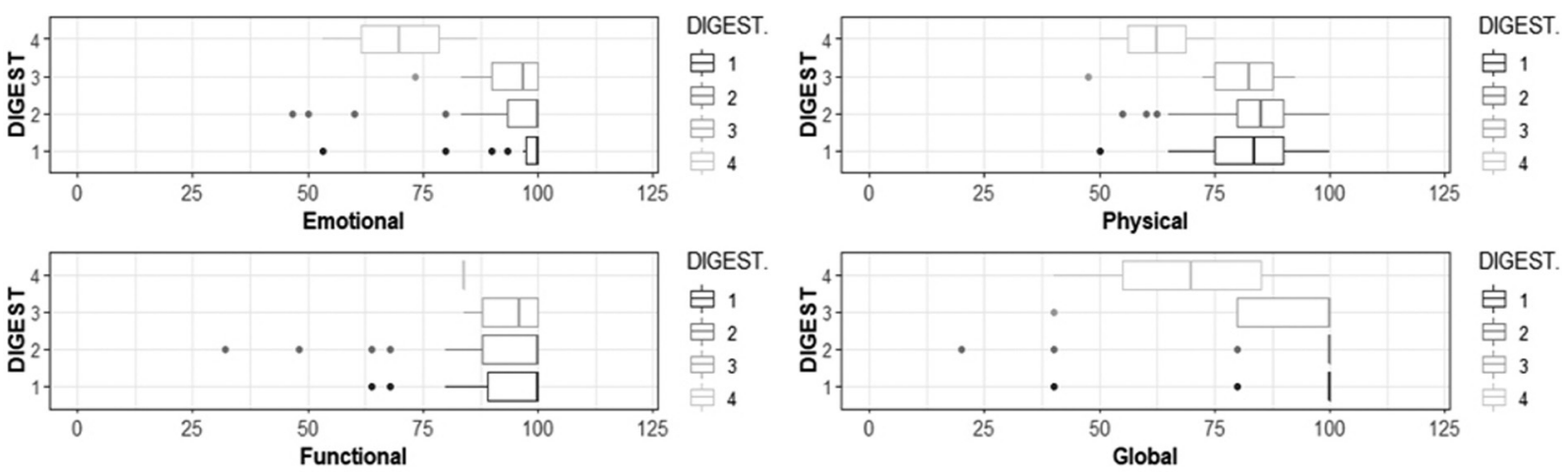

\section{DIGEST.}

官 1

官 2

官 3
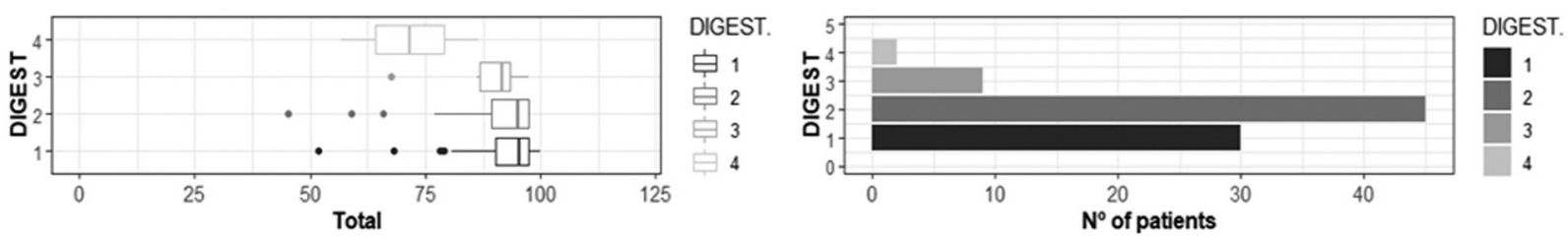

Fig. 2 Boxplot of the distribution by domain of the Deglutition Quality of Life Questionnaire M. D. Anderson Dysfaghia Inventory (MDADI) in relation to each classification from the Dynamic Imaging Grade of Swallowing Toxicity (DIGEST).

\section{Discussion}

The aim of the present study was to investigate the possible association between self-reported QoL and the objective assessment of swallowing performed by VFSS and to test, for this purpose, the use of two scales, PAS and DIGEST, in patients undergoing SCL-CHEP.

There was a prevalence of males, married, with low education, smokers and alcoholics at diagnosis and with intermediate or advanced stage laryngeal tumors of histopathological squamous cell carcinoma, a group consistent with the population commonly described for head and neck cancer. ${ }^{1-4,7,8}$

In patients with head and neck cancer, swallowing disorders are common, caused by the development of the disease in anatomical sites such as the oral cavity, the pharynx and the larynx, or by the treatment of adjacent structures. ${ }^{21}$ The investigation of QoL in swallowing makes it possible to identify the damages that dysphagia can cause in activities of daily living, mood, social limitations, and enable the best therapeutic intervention. , $3,9,21$ Quality of life protocols are tools that assist clinicians in building an individualized and contextualized rehabilitation. ${ }^{22}$

At the time of the clinical evaluation of swallowing, all patients had exclusive oral feeding and hydration. However, through the PAS and DIGEST scales, it is possible to understand some limitations in this function. In the classification on the DIGEST scale, there was a high prevalence of grades 1 and $2(87.2 \%)$ in the sample, suggesting that most patients had mild or moderate dysphagia. It is important to consider that there was no patient classified as DIGEST 0 (without dysphagia), making evident the characteristic of chronic dysphagia due to a surgical sequel, already described in 
the literature. ${ }^{7,9,13,21}$ Approximately 13\% of the individuals presented moderate or severe impairment of swallowing function, even at a late postsurgical moment, a fact that reinforces the long-term monitoring of patients. Swallowing may be influenced by other aspects, such as aging and several comorbidities. $^{2,8}$

In the clinical evaluation of swallowing using PAS, a higher prevalence of PAS 5 and 8 was observed, with a total of $82.54 \%$. The lowest frequency ratings were 4 and $2(n=15$; $17.43 \%)$. This data probably does not represent the reality of the QoL of the patients, due to PAS considering only penetration and aspiration in its severity classification. A different distribution was observed in the DIGEST, with a higher prevalence of more severe dysphagia. Supracricoid laryngectomy CHEP removes $\sim 70 \%$ of the organ, its resection and reconstruction promote important functional and structural changes that may lead to the formation of residues and impair the protection of the lower airways. ${ }^{5,7,9,13}$ In the long term, aspiration becomes chronic, and this functional adaptation reduces the impact on QoL due to its silent characteristic. ${ }^{9}$ This fact agrees with the large number of patients with good QoL in the MDADI questionnaire. ${ }^{3,9,23}$ There is a more severe group, with worse efficiency results, in which the presence of pharyngeal residue usually causes complaints of the sensation of stopped food, suffocation, and difficulty in progressing the diet. $7,14,15,21$ Therefore, it is possible to understand why the DIGEST results are more consistent with QoL scores.

Only three patients remained with a tracheostomy, an aspect that was not significant for this assessment of QoL; however, it is clear that it may affect their daily activities in some way. Tracheostomy has several social stigmas and requires daily management to maintain hygiene and minimize the risk of infection. ${ }^{24}$

More advanced cancer stages showed a significant relationship with the global and total domains in the MDADI questionnaire. This finding reinforces that early detection is the main way of maintaining the structures, the laryngeal functions and the QoL of patients with head and neck cancer. ${ }^{2,25}$

The main objective of cancer treatment is the control of the tumor with the maintenance of functions and, in the case of the larynx, the organ responsible for breathing, swallowing and speech, reaching this goal is a challenge. ${ }^{5,6,9,13,21}$ The radiotherapy protocols aim to conserve the organ; however, it is known that the preservation of function is not always achieved, especially in cases of advanced tumors, causing a negative impact on QoL. ${ }^{11}$ Partial laryngectomies aim to be an option for the treatment of moderately advanced tumors, without the permanence of permanent tracheostomia and preservation of the laryngeal voice and oral feeding. $1,5,6,9,13$ However, the maintenance of these functions is not complete and, in the case of swallowing, the patient remains with chronic dysphagia due to the removal of important structures for the protection of the lower airways. ${ }^{7,13}$ Even so, after a few months of therapy, most patients return to feeding exclusively via the oral route. ${ }^{7,9}$ Dysphagia is expected to have some impact on the patient, affecting his QoL. 3,23
The assessment of QoLin swallowing presented medians of values $>80$ in all domains. This result demonstrates that the proper selection and management of patients can generate adequate QoLstandards. ${ }^{2,3,9}$ The average MDADI score for the total domain reported in the literature ranges from 80 to 92 , demonstrating a good QoL in this group of patients. ${ }^{2,9}$ In a recent study, Pizzorni et al. investigated QoL related to swallowing after SCL-CHEP .and reported that it was significantly affected by the severity of aspiration, with higher mean scores on the functional subscale of the MDADI questionnaire and lower scores on the physical subscale. ${ }^{9}$ Similar results were observed, with an average total score of 89.33 and with the same distribution of scores in the subscales, suggesting a long-term QoL.

For a group that is known to have chronic dysphagia, the positive homogeneity of QoL results leads to consider that there is still a need for tools that consider other aspects of swallowing dynamics. Other studies have already shown that residue has a negative impact on the lives of patients with head and neck cancer, and the identification of an instrument that considers this parameter is essential ${ }^{14}$ to improve the assessment and rehabilitation.

Lower averages of MDADI values are consistent with worse swallowing values attributed by DIGEST, which, despite not having been statistically significant, shows adequate behavior compared with that observed in the clinic. The results also demonstrated a negative correlation between the DIGEST and the emotional domain of the QoL protocol, reinforcing the hypothesis that the efficiency of swallowing could have a relevant impact on the QoL of these individuals. ${ }^{9,14}$

The present study presents a limitation in the reduced number of patients. Further future assessments with an expanded group in more than one center are required. It is necessary to find ways of assessing functional changes that are in agreement with the clinical status and QoL perceptions of the individuals. The result of the functional scales needs to contemplate the patient in a multidimensional way to enable assertive interventions. The swallowing function is of great importance for the maintenance of the life and well-being of the individual; there is no coherence when we describe a negative finding of a function and there are no impacts on QoL. It is evident that some aspect is not being contemplated.

In the present study, PAS did not show agreement with QoL. The most severe PAS did not necessarily represent worse QoL values. It is possible to observe a QoL progression behavior according to the best performance on the DIGEST swallowing scale. Residue was a more relevant aspect for QoL, making the DIGEST a promising tool in the assessment of dysphagic patients.

\section{Conclusion}

The DIGEST and PAS did not obtain similar results to assess the swallowing of patients undergoing supracricoid laryngectomy. The PAS classified swallowing with the highest severity rates. The DIGEST was more consistent with the QoL assessment. More advanced oncology stays had a significant 
relationship with the global and total domains in the MDADI questionnaire. Medians $>80$ were observed in all domains of the MDADI questionnaire, The appropriate selection and management of patients can generate adequate QOL standards.

There was a reduction in the mean value of MDADI that accompanies the worsening of the swallowing function attributed by the DIGEST and a negative correlation between the DIGEST and the emotional domain of the QoL protocol, reinforcing the hypothesis that swallowing efficiency has a more relevant impact on QoL than just safety.

\section{Conflict of Interests}

The authors have no conflict of interests to declare.

\section{References}

1 Schindler A, Pizzorni N, Mozzanica F, et al. Functional outcomes after supracricoid laryngectomy: what do we not know and what do we need to know? Eur Arch Otorhinolaryngol 2016;273(11): 3459-3475

2 Zica GM, Freitas AS, Silva AC, et al. Deglutição, voz e qualidade de vida de pacientes submetidos à laringectomia supratraqueal alargada. Einstein (Sao Paulo) 2020;18:eAO5390

3 Di Santo D, Bondi S, Giordano L, et al. Long-term Swallowing Function, Pulmonary Complications, and Quality of Life after Supracricoid Laryngectomy. Otolaryngol Head Neck Surg 2019; 161(02):307-314

4 Succo G, Crosetti E. Limitations and Opportunities in Open Laryngeal Organ Preservation Surgery: Current Role of OPHLs. Front Oncol 2019;9:1-8

5 Majer H, Rieder W. Technic of laryngectomy permitting the conservation of respiratory permeability (cricohyoidopexy). Ann Otolaryngol Jul-Aug1959;76:677-681

6 Laccourreye H, Laccourreye O, Weinstein G, Menard M, Brasnu D. Supracricoid laryngectomy with cricohyoidoepiglottopexy: a partial laryngeal procedure for glottic carcinoma. Ann Otol Rhinol Laryngol 1990;99(6 Pt 1):421-426

7 Pizzorni N, Schindler A, Castellari M, Fantini M, Crosetti E, Succo G. Swallowing Safety and Efficiency after Open Partial Horizontal Laryngectomy: A Videofluoroscopic Study. Cancers (Basel) 2019; 11(04):549

8 Schindler A, Pizzorni N, Fantini M, et al. Long-term functional results after open partial horizontal laryngectomy type IIa and type IIIa: A comparison study. Head Neck 2016;38(01, Suppl 1): E1427-E1435

9 Pizzorni N, Schindler A, Fantini M, et al. Relationship between swallowing-related quality of life and fiberoptic endoscopic evaluation of swallowing in patients who underwent open partial horizontal laryngectomy. Eur Arch Otorhinolaryngol 2018;275 (04):973-985
10 Tribius S, Meyer MS, Pflug C, et al. Socioeconomic status and quality of life in patients with locally advanced head and neck cancer. Strahlenther Onkol 2018;194(08):737-749

11 Tribius S, Raguse M, Voigt C, et al. Residual deficits in quality of life one year after intensity-modulated radiotherapy for patients with locally advanced head and neck cancer: Results of a prospective study. Strahlenther Onkol 2015;191(06):501-510

12 Rosenbek JC, Robbins JA, Roecker EB, Coyle JL, Wood JL. A penetration-aspiration scale. Dysphagia 1996;11(02):93-98

13 Lips M, Speyer R, Zumach A, Kross KW, Kremer B. Supracricoid laryngectomy and dysphagia: A systematic literature review. Laryngoscope 2015;125(09):2143-2156

14 Meyer TK, Pisegna JM, Krisciunas GP, Pauloski BR, Langmore SE. Residue influences quality of life independently of penetration and aspiration in head and neck cancer survivors. Laryngoscope 2017;127(07):1615-1621

15 Hutcheson KA, Barrow MP, Barringer DA, et al. Dynamic Imaging Grade of Swallowing Toxicity (DIGEST): Scale development and validation. Cancer 2017;123(01):62-70

16 Pisegna JM, Kaneoka A, Leonard R, Langmore SE. Rethinking Residue: Determining the Perceptual Continuum of Residue on FEES to Enable Better Measurement. Dysphagia 2018;33(01):100-108

17 Steele CM, Peladeau-Pigeon M, Nagy A, Waito AA. Measurement of Pharyngeal Residue From Lateral View Videofluoroscopic Images. J Speech Lang Hear Res 2020;63(05):1404-1415

18 Logemann JA. The evaluation and treatment of swallowing disorders. Current Opinion in Otolaryngology. Head Neck Surg 1998; 6(06):395-400

19 Chen AY, Frankowski R, Bishop-Leone J, et al. The development and validation of a dysphagia-specific quality-of-life questionnaire for patients with head and neck cancer: the M. D. Anderson dysphagia inventory. Arch Otolaryngol Head Neck Surg 2001;127 (07):870-876

20 Guedes RL, Angelis EC, Chen AY, Kowalski LP, Vartanian JG. Validation and application of the M.D. Anderson Dysphagia Inventory in patients treated for head and neck cancer in Brazil. Dysphagia 2013;28(01):24-32

21 Zica GM, Freitas AS. Deliberações clínicas na atuação em pacientes com câncer de cabeça e pescoço: atualidades e desafios na disfagia. Distúrb Comun 2019;31(04):693-697

22 Maghami E, Ismaila N, Alvarez A, et al. Diagnosis and Management of Squamous Cell Carcinoma of Unknown Primary in the Head and Neck: ASCO Guideline. J Clin Oncol 2020;38(22):2570-2596

23 Topaloglu I, Köprücü G, Bal M. Analysis of swallowing function after supracricoid laryngectomy with cricohyoidopexy. Otolaryngol Head Neck Surg 2012;146(03):412-418

24 Kutsukutsa J, Kuupiel D, Monori-Kiss A, Del Rey-Puech P, MashambaThompson TP. Tracheostomy decannulation methods and procedures for assessing readiness for decannulation in adults: a systematic scoping review. Int J Evid-Based Healthc 2019;17(02):74-91

25 Bonzanini LIL, Soldera EB, Ortigara GB, et al. Clinical and sociodemographic factors that affect the quality of life of survivors of head and neck cancer. Support Care Cancer 2020;28(04):1941-1950 